\title{
CAMINOS DE RENOVACIÓN A PARTIR DE LA TRADICIÓN: LA OBRA DEL ICONÓGRAFO GRIEGO MICHALIS VASILAKIS
}

\author{
Federico Aguirre \\ Facultad de Teología \\ Pontificia Universidad Católica de Chile
}

Resumen: El presente artículo tiene como objetivo presentar la obra del pintor iconógrafo griego Michalis Vasilakis. La obra de Vasilakis constituye una de las expresiones más intrépidas de la pintura de íconos contemporánea en Grecia, incorporándose a la milenaria trayectoria de la tradición en cuestión y ganando a su vez un merecido reconocimiento en la escena artística neohelena. Nuestra presentación se organiza del siguiente modo: en primer lugar realizamos una breve reseña histórica sobre el renacimiento de la pintura de íconos en Grecia moderna para luego proceder a la presentación y el análisis de la vida y obra del artista.

Palabras clave: pintura de íconos - Michalis Vasilakis - arte griego contemporáneo.

\section{PATHS TO RENEWAL FROM TRADITION: THE WORK OF THE GREEK ICONOGRAPHER MICHALIS VASILAKIS}

Abstract: This article aims to present the work of the Greek icon painter Michalis Vasilakis. Vasilakis' work, one of the most intrepid expressions of contemporary icon painting in Greece, is incorporated in the age-old tradition in question and has achieved a well-deserved recognition in modern Greek artistic scene. Our presentation is organised in the following way: firstly, we provide a brief historical background of the rebirth of icon painting in modern Greece, so that we can then proceed to the presentation and analysis of the artist's life and work.

Keywords: Icon painting - Michalis Vasilakis - Contemporary Greek art.

Reecibido: 17.11.2019 - Aceptado: 30.04.2020

\section{Correspondencia: Federico Aguirre}

Email: federico.aguirre@uc.cl

Doctor en Estudios Lingüísticos, Literarios y Culturales

Profesor e investigador, Centro de Estudios de la Religión

Facultad de Teología, Universidad Católica de Chile 


\section{Introducción: la recuperación de la tradición del ícono en el contexto de Grecia moderna.}

1 interés por la denominada "pintura bizantina" aumenta
exponencialmente desde su descubrimiento a mediados del
siglo XIX. La investigación iniciada por el arqueólogo francés Adolphe Didron en 1839, a partir del siglo XX se convierte en disciplina autónoma bajo el nombre de "bizantinología", disciplina hoy presente en el currículo de prestigiosas universidades en todo el mundo. En este contexto y como destaca el historiador alemán Hans Belting (Belting: 2009), la tradición pictórica de Bizancio ha sido restituida en el ámbito de la historia del arte como un eslabón perdido entre el arte de la Antigüedad tardía y el arte del Renacimiento. No obstante, la fascinación por Bizancio no se limita a un interés académico. Actualmente en todo el mundo proliferan talleres donde se enseñan las técnicas tradicionales de la pintura de íconos y se profundiza en su sentido teológico. Por su parte, en países de tradición ortodoxa como Rusia y Grecia, el redescubrimiento de la tradición pictórica de Bizancio jugará un rol fundamental en la conformación de las Vanguardias artísticas de principios del siglo $\mathrm{XX}$, dando lugar a una síntesis entre tradición y modernidad. Así pues, en el ámbito griego en particular, a partir de mediados del siglo XX la tradición pictórica del ícono será restituida como práctica actual, convirtiéndose hoy en plataforma de interesantes propuestas artísticas. En este ámbito, pues, donde lo antiguo y lo nuevo se conjugan como un presente cargado de sentido, se inscribe la obra del pintor griego contemporáneo Michalis Vasilakis.

Antes de abocarnos a Vasilakis, haremos una breve reseña del proceso de restitución de la tradición del ícono en el contexto de Grecia moderna, con el objeto de situarnos en una perspectiva adecuada para la valoración de su obra. Como es sabido, a partir del Renacimiento y con la aparición de la pintura naturalista europea, la tradición del ícono se fue marginando progresivamente, hasta ser considerada durante el periodo de la Ilustración como un arte bárbaro. Entre los promotores de esta opinión, se encuentra nada más y nada menos que el padre de la historiografía moderna, Edward Gibbon, en cuya obra el juicio de Bizancio es tajante (Gibbon: 1993). En el ámbito cultural griego, desmembrado después de la caída de Constantinopla a manos de los turcos otomanos en 1453, el arte del ícono irá cediendo terreno a la influencia de Occidente, fenómeno presente principalmente en centros urbanos bajo dominio veneciano, tales como Heraclión, en Creta, y otras ciudades de las islas del mar jónico. 
El resultado de este proceso será la formulación progresiva de un estilo bizantino "mejorado", que a partir de la liberación de los turcos y la formación del Estado griego moderno en 1832 se convertirá en el estilo oficial de la Iglesia ortodoxa griega.

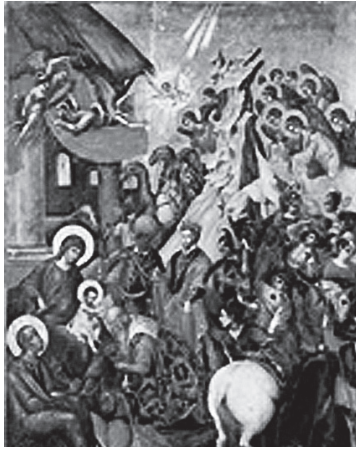

1. M. Damaskinós, $L a$ adoración de los magos, s. XVII. (Representante de la denominada "escuela cretense").

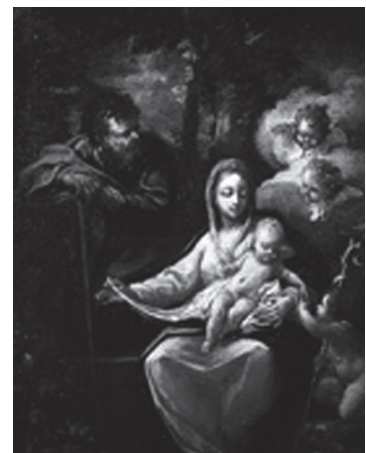

2. P. Doxarás, La sagrada familia, s. XVIII.

(Representante de la pintura de las islas del Mar Jónico)

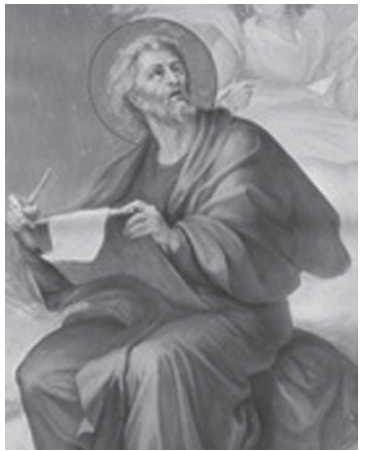

3. K. Artémis, san Marcos, finales s. XIX. (Representante de la pintura "oficial" de la Iglesia ortodoxa griega del s. XIX)

De este modo, durante el siglo XIX, el arte del ícono será sustituido por un estilo de corte naturalista completamente ajeno a la tradición plástica bizantina. No obstante, durante este periodo de casi 100 años en que la pintura bizantina desaparece de las iglesias del nuevo Estado, la tradición del ícono pervivirá vinculada a la cotidianidad del pueblo griego en zonas todavía bajo ocupación turca como Asia menor y algunas islas del mar Egeo, cunas milenarias de la cultura helena pero todavía desvinculadas del nuevo Estado. Y esta tradición no pervivirá sólo en las Iglesias sino también en diferentes ámbitos de la vida civil, dando lugar a un arte denominado "popular" que se convertirá en sustrato del arte griego moderno. Así, por paradójico que parezca, los pintores griegos que introducen el arte moderno en Grecia recurrirán a esta tradición bizantino-popular para llevar a cabo su proyecto de modernidad, dado que en esta tradición reconocerán una Vanguardia avant la lettre. Uno de los estandartes de este nuevo "helenismo" será la obra del pintor autodidacta Theóphilos (1870-1934), la cual, además de inspirar a poetas y pintores de la Vanguardia griega, será expuesta en París como paradigma del espíritu moderno. 


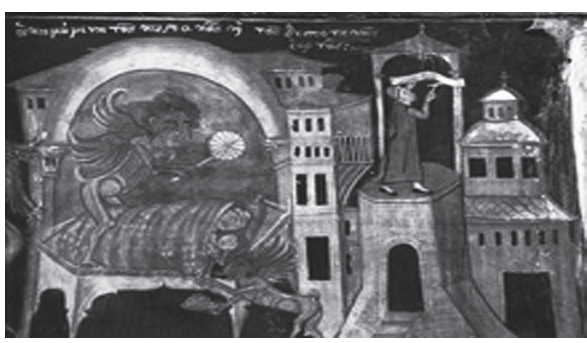

4. Pagoni, Escenas del infierno, Iglesia de Santa Marina, Pilio, 1802.

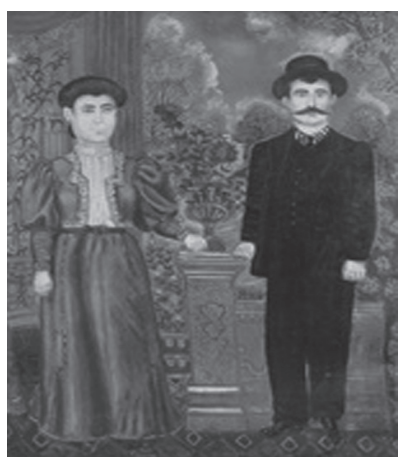

5. Theóphilos, Pareja, 1919.

Entre otros artistas griegos que introducen las corrientes del arte moderno en Grecia y al mismo tiempo buscan en la tradición bizantina un referente formal, cabe destacar la obra de Phótis Kóntoglou (1895-1965). Kóntoglou, después de una estancia en París y otros países de Europa occidental donde entra en contacto con los principales movimientos del arte moderno, regresa a Grecia y descubre en la pintura bizantina un camino propiamente griego hacia la modernidad. En 1927 visita el Monte Athos y en 1932 los monasterios de Metéora, donde se maravillará con los frescos bizantinos y las colecciones de íconos portables, y se entregará al estudio de las técnicas tradicionales con el objeto de comprender la particularidad plástica de este arte milenario. Así, a partir de la década del 30, la obra de Kóntoglou sintetizará la sabiduría plástica del ícono con las búsquedas del arte moderno. Uno de los frutos de esta labor serán las pinturas murales del Ayuntamiento de Atenas realizadas entre 1935 y 1937, obra donde Kóntoglou cifrará su propuesta de un arte propiamente griego y eminentemente moderno.

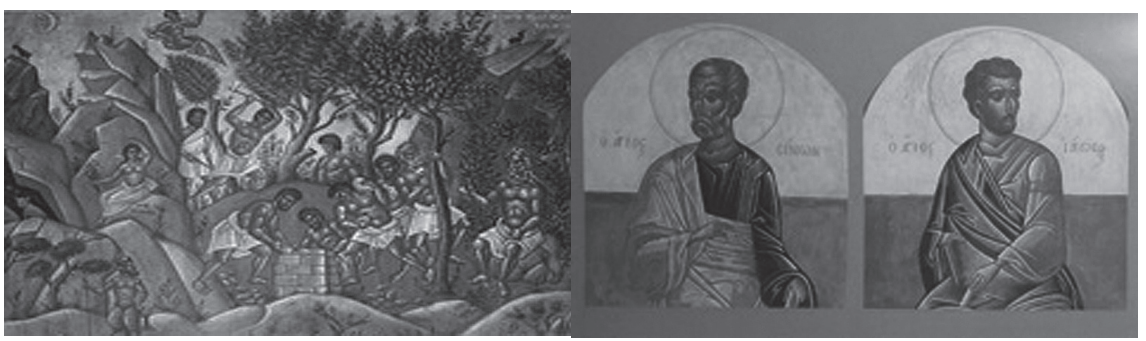

6. Ph. Kontoglou, Las hazañas de Teseo, Ayuntamiento de Atenas, 1935-7.
7. Ph. Kontoglou, apóstoles Simón y Jaime, iconostasio de la capilla de la Cruz Roja de Atenas, 1937. 
Ahora bien, a diferencia de otros artistas de su época que se entregan al estudio del arte bizantino, Kóntoglou no buscará sólo referencias formales sino que se propondrá restituir la tradición del ícono como práctica contemporánea. Así, desde finales de la década del 40 el artista griego se abocará exclusivamente al trabajo de restauración y a la ornamentación de templos ortodoxos. A la luz de esta experiencia, elaborará un completo manual técnico e iconográfico que compendia los conocimientos necesarios para la labor del iconógrafo, volumen que será publicado en 1960 bajo el título de Ékphrasis tis orthodóxou eikonographías y que será premiado por la Academia de Letras de Atenas. De este modo, Kóntoglou se convertirá en el principal promotor de la restitución de la tradición del ícono en Grecia, instituyéndose por ley del estado el denominado estilo "neobizantino" como estilo oficial de las Iglesias ortodoxas en territorio griego a partir de finales de los años 30. Es importante destacar que dicha restitución no está exenta de conflictos, en los cuales, sin embargo, no podemos profundizar aquí. Lo que nos interesa subrayar es que, a partir de la segunda mitad del siglo XX y gracias a la semilla plantada por Kóntoglou, la obra iconográfica de artistas griegos como Vasilakis encontrará un camino para vincular la tradición milenaria del ícono a la problemática estética contemporánea.

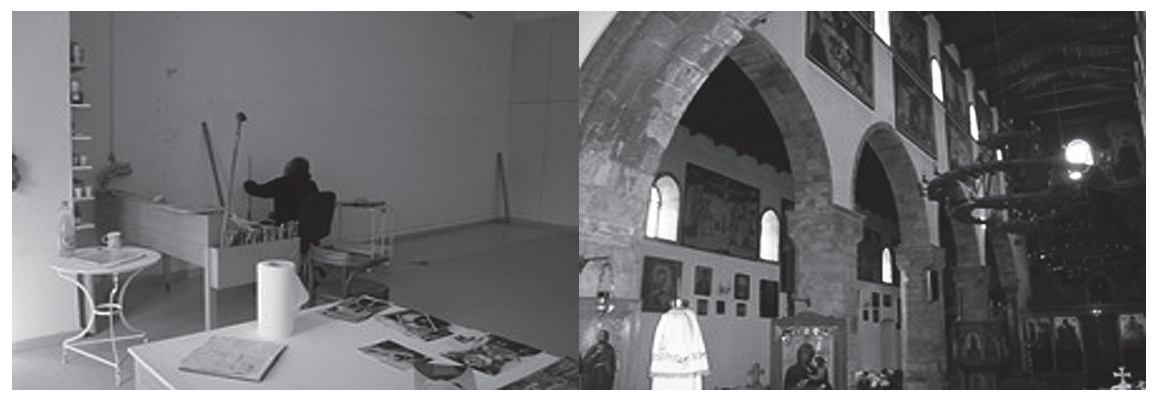

8. Michalis Vasilakis en su taller, Heraclión, Creta, 2011.
9. Iglesia de la Virgen de los Cruzados, Heraclión, Creta. 


\section{La Virgen de los Cruzados: el taller de experimentación.}

Michalis Vasilakis nace en Anópolis, Creta, en 1948. A los 20 años comienza a trabajar como ayudante del iconógrafo Manólis Betinákis pintando diversas Iglesias de la isla de Creta. Como Kóntoglou, Vasilakis también realizará un periplo por Europa occidental durante la década de los 70, estableciéndose finalmente en la capital de Creta, Heraclión, donde fija su taller dedicándose por más de 30 años al oficio de la iconografía. Para aproximarnos a la poética del artista cretense, presentaremos obras de tres conjuntos iconográficos, los cuales muestran claramente el itinerario de Vasilakis desde una iconografía más bien convencional a una propuesta plástica basada en la abstracción.

El primero de estos conjuntos se encuentra albergado en la Iglesia $\mathrm{La}$ Virgen de los Cruzados, ubicada en el centro de Heraclión. En esta Iglesia se albergan obras de diferentes periodos, desde la década de los 80 hasta principios del 2000, por lo que nos servirá para obtener una panorámica del itinerario plástico del artista griego. El primer estadio de este itinerario, como hemos destacado, viene marcado por la imitación de prototipos del pasado, fenómeno bastante frecuente en el ámbito de la iconografía a partir de mediados del siglo XX. Así, en la representación del Descenso a los infiernos de Vasilakis (fig. 10), obra de principios de los 80, podemos reconocer una referencia casi literal a la misma escena de la Iglesia de San Salvador de Chora, en Estambul, obra maestra del siglo XIV (fig. 11).

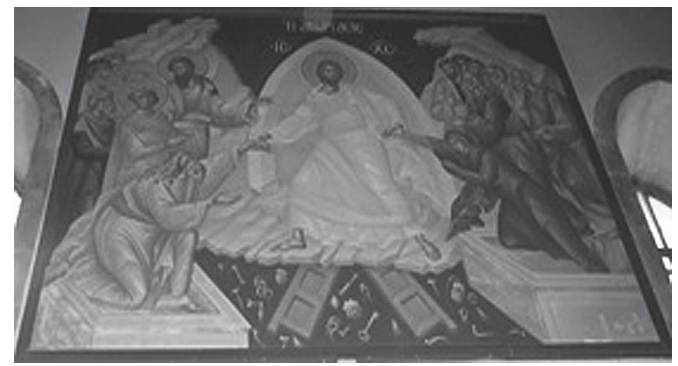

10. M. Vasilakis, El descenso a los infiernos, 1982.

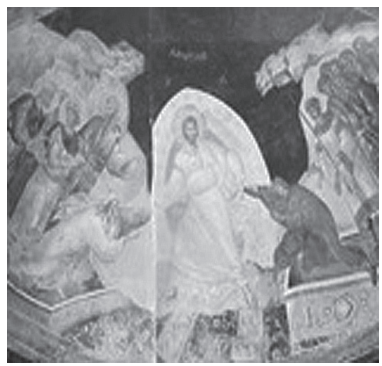

11. El descenso a los infiernos, San Salvador de Chora, Estambul, s. XIV. 
Ahora bien, la imitación de modelos del pasado parecería contradecir el espíritu de renovación inaugurado por Kóntoglou y la posibilidad de un diálogo con el arte de nuestro tiempo, y en alguna medida así es. No obstante, en el caso de Vasilakis, la imitación constituirá sólo un estadio de su producción iconográfica, cuyo sentido viene dado por un proceso de conocimiento y apropiación de la tradición. En este sentido, a finales de los 80 ya se puede apreciar una lectura más creativa de los prototipos. En los íconos del iconostasio de la Virgen de los cruzados, si bien reconocemos los prototipos de Teófanes el Cretense (maestro iconógrafo del siglo XVI), se puede observar una tendencia más selectiva (fig. 12 y 13); es decir, mientras que en el caso del Descenso a los infiernos casi no existe mediación, en el caso de los santos del iconostasio se observa claramente una lectura del modelo, donde se han simplificado una serie de elementos de la composición. Así pues, como reconoce el mismo Vasilakis, la denominada "escuela cretense" suscitará en él la búsqueda de una lógica de abstracción en la tradición pictórica bizantina.

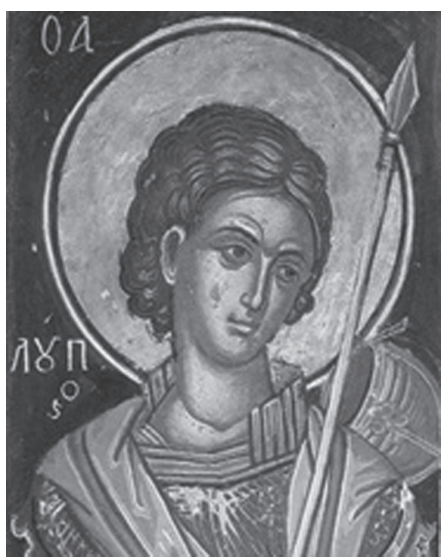

12. Teófanes el Cretense, San Loupos, s. XV.

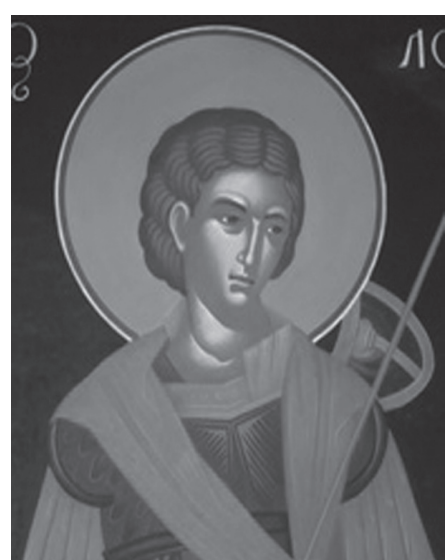

13. M. Vasilakis, San Loupos, finales $80^{\prime}$ 
De este modo, durante la década de los 90, Vasilakis probará diferentes grados de abstracción tanto a nivel cromático como compositivo. Testimonio de esta búsqueda son las obras que representan el Himno Akathistos a la Virgen María, serie ubicada en la nave central de la Virgen de los cruzados y donde podemos observar un particular tratamiento de las formas arquitectónicas y el paisaje (fig. 14 y 15). Para apreciar mejor este estadio donde Vasilakis plantea un primer "pensamiento plástico" personal, procederemos a presentar el segundo templo que hemos escogido para nuestro análisis. En cualquier caso, cabe destacar que a partir de este momento Vasilakis se dará cuenta de que la única manera de hacer pervivir una tradición es haciéndola caminar a través de una búsqueda personal; como destaca el mismo artista en una entrevista del año 1998, la tradición no existe sencillamente para ser conservada y admirada en los museos, sino sobre todo para mostrarnos caminos de creación.

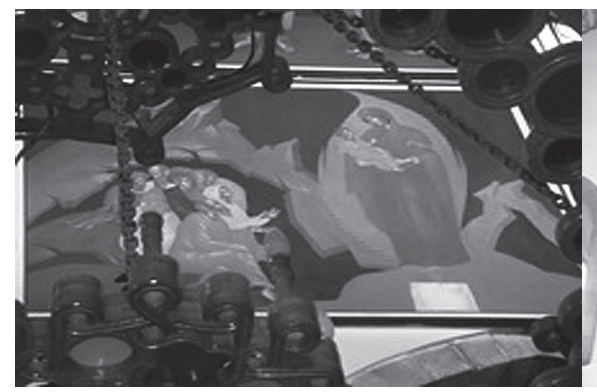

14. M. Vasilakis, Akáthistos Hymnos tis Panagías, 1992-4

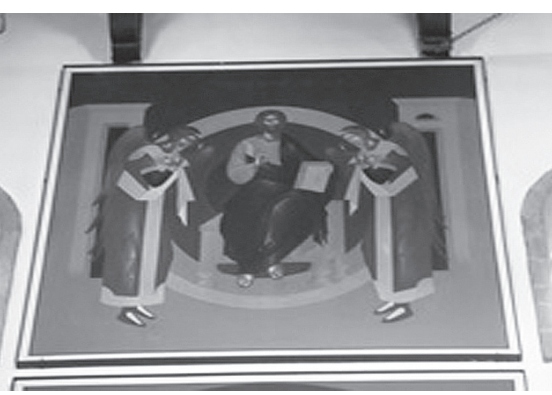

15. M. Vasilakis, Akáthistos Hymnos tis Panagias, 1992-4

\section{Santa Paraskeví: la concepción de un pensamiento plástico.}

La Iglesia de Santa Paraskeví es un pequeño templo de una sola nave que se ubica en el corazón de Heraclión. Se trata de una construcción sumamente sencilla en plena armonía con la tendencia minimalista del artista griego. Como apreciamos en la imagen número 16, la intervención de Vasilakis se concentra en la bóveda y está constituida por 6 medallones ubicados en la parte superior y la representación de las 12 fiestas de la ortodoxia o Dodekáorto en los laterales; en el fondo de la pequeña iglesia, podemos atisbar el ícono de Pentecostés. En los medallones situados en la parte superior de la bóveda (fig. 18 y 19) reconocemos una aplicación más consciente de ciertos elementos de abstracción observados por Vasilakis en 
las obras de la "escuela cretense". Así, Vasilakis observa en dicho periodo de la pintura bizantina una inclinación a definir los rasgos de la cara a través de manchas de color que se diferencian y destacan claramente contra el fondo café del rostro, evitando una transición tonal. Este tratamiento de las zonas del cuerpo será el punto de partida del camino de Vasilakis hacia la abstracción, elemento que observamos ya en las obras de la década de los 80. También vale la pena destacar el tratamiento plano que presenta la mantilla de la Virgen, donde han desaparecido los pliegues para dar lugar a un achurado que destaca contra un fondo plateado.

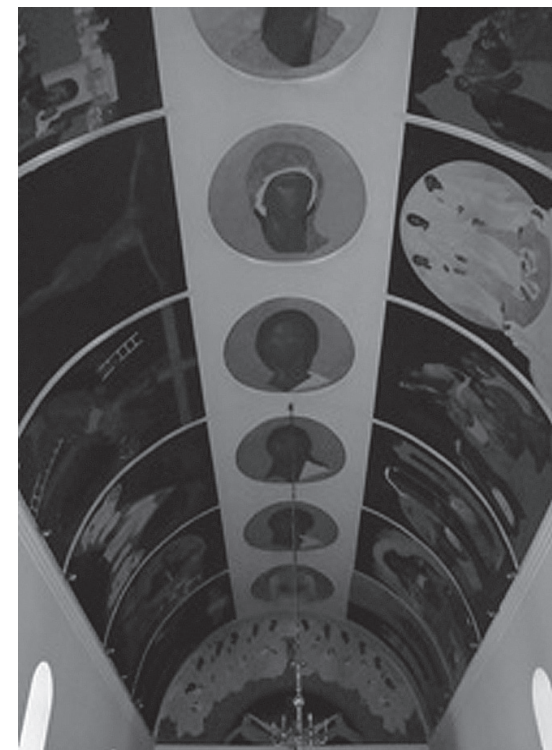

16. Iglesia de santa Paraskeví, Heraclión, Creta.

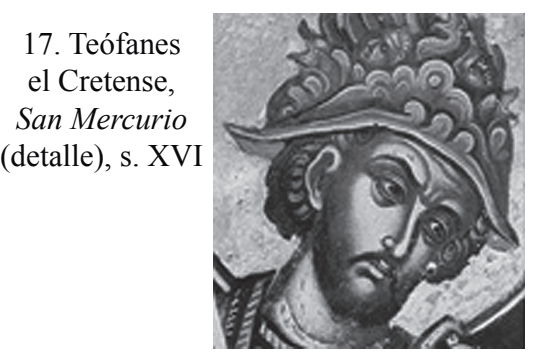

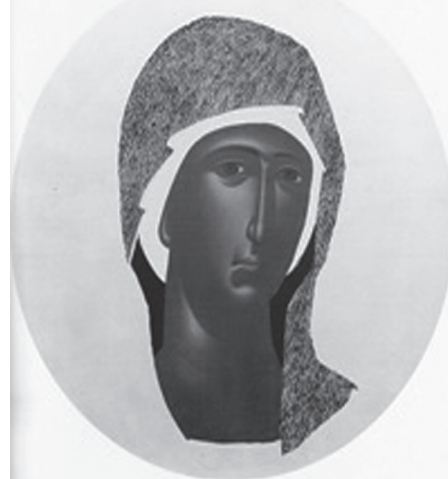

18. M. Vasilakis, Madre de Dios, 1992-4.

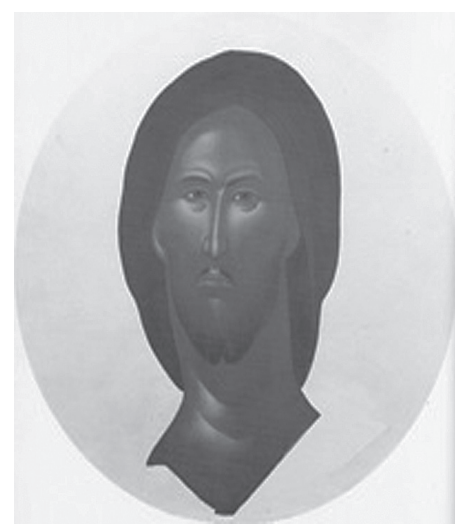

19. M. Vasilakis, Jesucristo Pantocrátor, 1992-4. 
Ahora bien, la búsqueda de Vasilakis no se limitará a la "escuela cretense". En el tratamiento de las vestimentas apreciamos una clara influencia de la obra de Teófanes el Griego, el padre de la iconografía rusa, que conoce su acmé durante los siglos XV y XVI. Así, según Vasilakis la "escuela rusa" es prima hermana de la "escuela cretense", en tanto que comparten una especial inclinación a la abstracción. Como podemos apreciar en las figuras 20 y 21, Vasilakis reproduce la operación que Teófanes el Griego lleva a cabo para otorgar mayor dinamismo y expresividad a los cuerpos, la cual consiste en la abstracción de los tonos intermedios y la iluminación puntual de las zonas del cuerpo que concentran el movimiento.

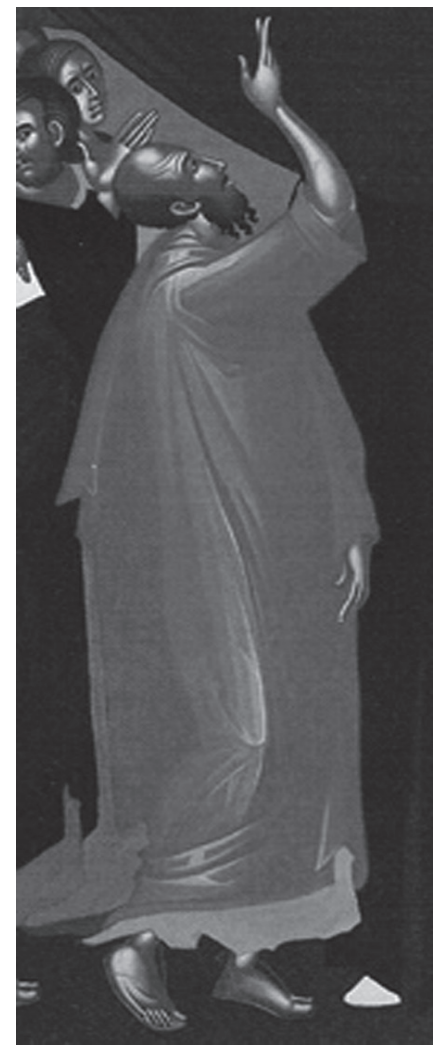

20. M. Vasilakis, La Ascención de la Virgen (detalle), 1992-4.

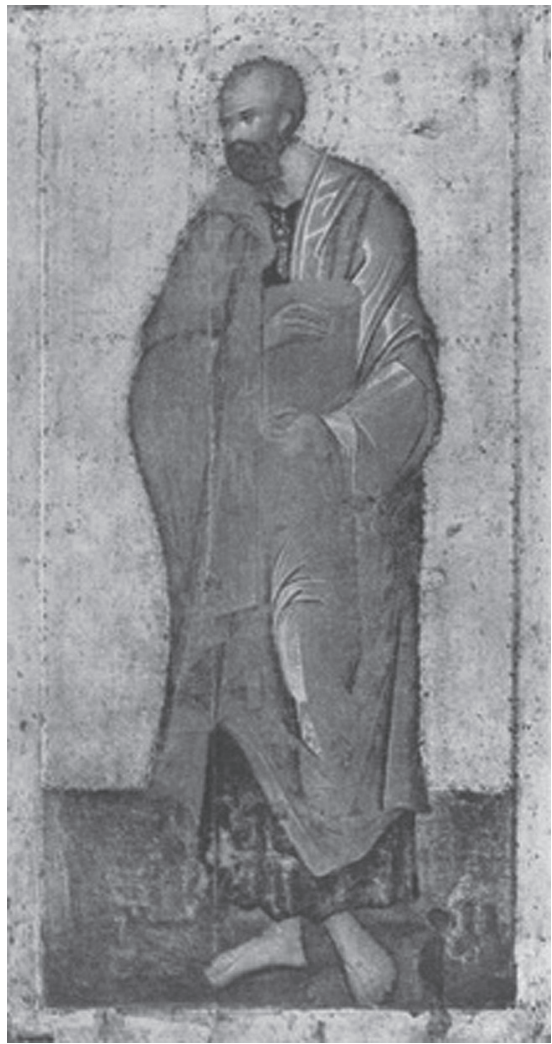

21. Teófanes el Griego, Apóstol Pablo, s. XV. 
No obstante, durante este estadio de su obra, la aplicación más osada de la abstracción por parte de Vasilakis se observa en el tratamiento de la arquitectura y el paisaje. En las imágenes del Dodekáorto podemos observar un tratamiento similar de la arquitectura y el paisaje al observado en el último conjunto de la Virgen de los cruzados. Ambos conjuntos pertenecen al mismo periodo, y se caracterizan por una clara disposición geométrica de los elementos, subrayada por fuertes contrastes cromáticos. Esta aplicación de la abstracción geométrica, como hemos visto, todavía se muestra tímidamente, y aún más en la representación de la figura humana, donde se reconoce un tratamiento en alguna medida más naturalista que en el caso de la arquitectura y el paisaje.

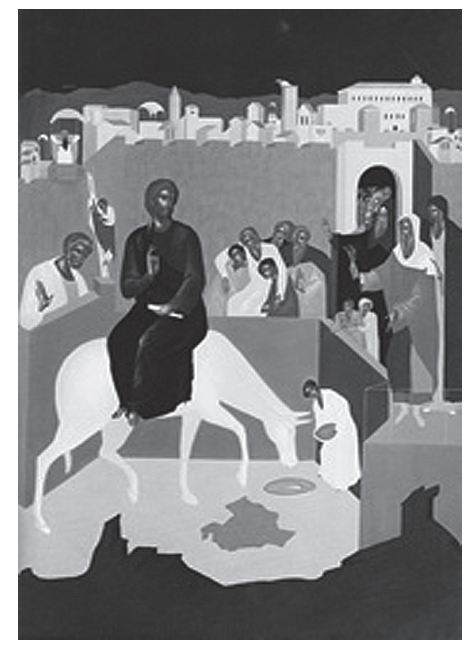

22. M. Vasilakis, La entrada a Jerusalén, 1992-4.

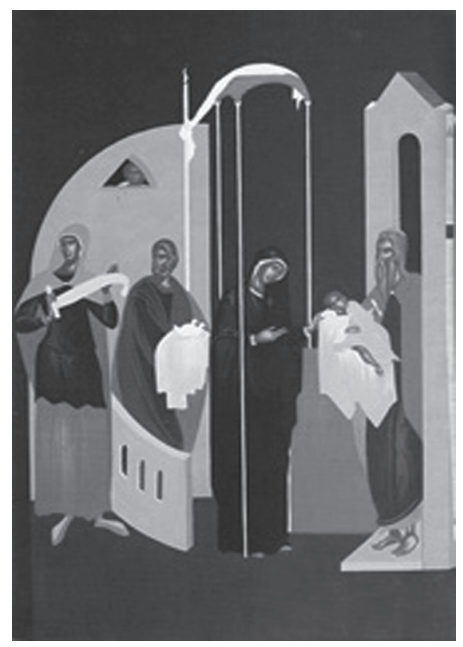

23. M. Vasilakis, La presentación en el templo, 1992-4.

Así, hasta aquí podríamos hablar de dos tendencias en la obra de Vasilakis: por una parte, la aplicación libre de la geometría en las formas arquitectónicas y el paisaje, las cuales se representan a través de superficies planas y, por otra, en el caso de la figura humana, la búsqueda de ciertos elementos de la tradición bizantina en los cuales el artista griego reconoce una lógica de abstracción. La síntesis de estas dos tendencias tendrá lugar a partir de la próxima década, en las obras que veremos a continuación. Cabe subrayar que esta elaboración de una lógica de abstracción por parte de Vasilakis no surge de un simple capricho sino del reconocimiento de un 
elemento de la tradición que, según el artista griego, se hace especialmente elocuente en nuestros días. En este sentido, las obras de Santa Paraskeví conocerán una excelente recepción por parte de la prensa y destacadas personalidades del mundo cultural griego como el reconocido bizantinista Manólis Chatzidákis.

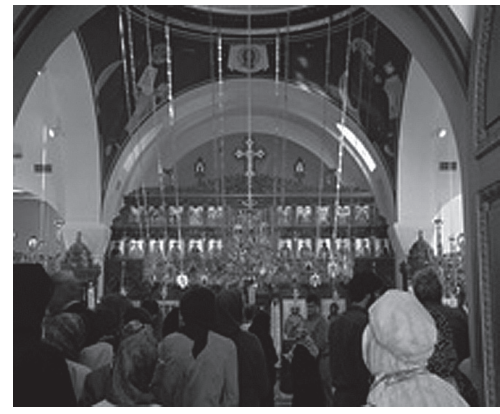

24. Iglesia del Monasterio Zoodochos Pigis, San Francisco, EE.UU.

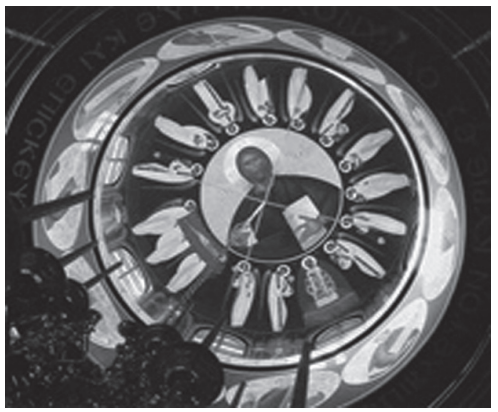

25. Iglesia del Monasterio Zoodochos Pigís, San Francisco, EE.UU.

\section{Monasterio Zoodóchos Pigí:}

\section{la concreción de la propuesta plástica de M. Vasilakis.}

Las obras encargadas a Vasilakis a finales de los 90 para la Iglesia del Monasterio Zoodóchos Pigí en San Francisco, EE.UU, se constituirán en plataforma para la consumación de la propuesta plástica del artista griego. Como veremos a continuación, aquella lógica de abstracción que aparece como tendencia, en este estadio de la obra de Vasilakis se convertirá en el principio rector de cada operación pictórica. Para realizar este paso, sin embargo, Vasilakis conjugará la sabiduría plástica de la tradición del ícono con elementos de la pintura abstracta de la segunda mitad del siglo XX, declarando su decidida voluntad de que dicha tradición siga caminando en nuestros días y hablando para nosotros. En esta última parte de nuestra presentación, nos concentraremos en el conjunto iconográfico del Dodekáorto, una serie de doce cuadros que representan la vida de Jesús. Cabe destacar que con este conjunto Vasilakis sellará su presencia en la escena artística griega contemporánea, a través de exposiciones realizadas en Creta y Atenas, la publicación de diversos artículos sobre su obra y entrevistas en televisión y prensa escrita. 


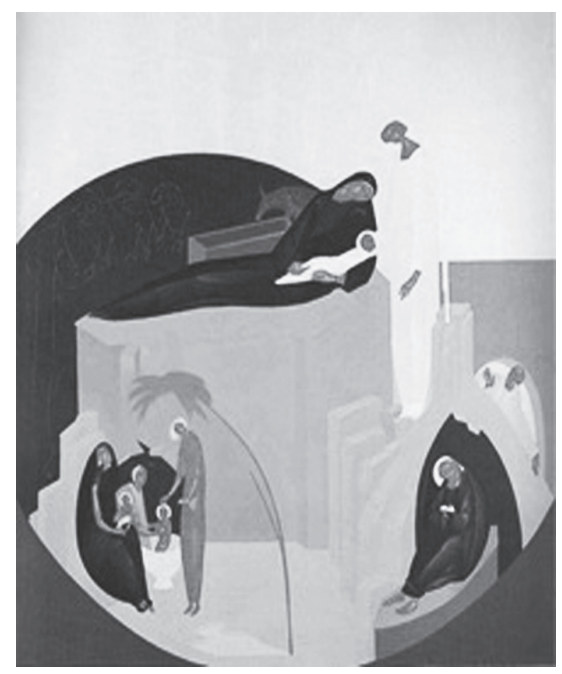

26. M. Vasilakis, La Natividad, 2002, $(1,85 \times 1,75 \mathrm{~m})$.

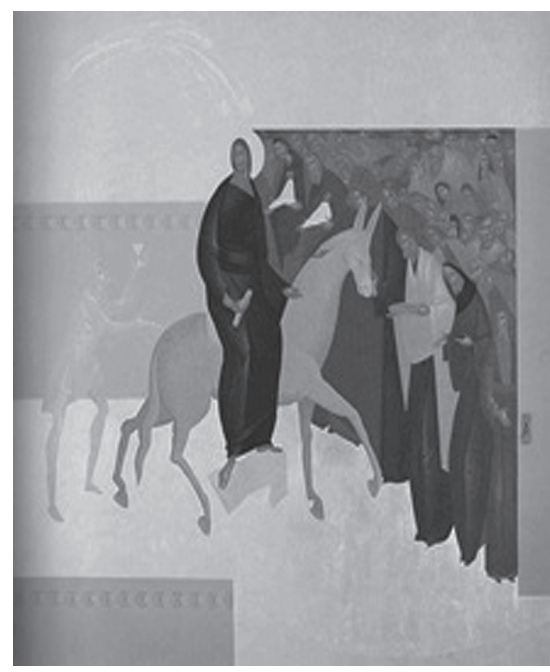

27. M. Vasilakis, La entrada a Jerusalén, $2002,(1,85 \times 1,75 \mathrm{~m})$.

Lo primero que destaca en esta serie, es la organización de la composición a partir de una rigurosa definición geométrica de la superficie pictórica, operación que Vasilakis aplica ya desde principios de la década de los 90 para el tratamiento de la arquitectura y el paisaje. En el conjunto que examinamos ahora, no obstante, se ha radicalizado dicha operación, cuyo resultado es el empleo de árboles, casas y cerros como figuras geométricas puras, haciéndose eco de la conocida máxima de Cézanne: abordar la naturaleza a través del cilindro, la esfera y el cono (Cézanne: 371). Como se hace perceptible en las figuras 26 y 27, el resultado de esta unificación geométrica del fondo con el paisaje y la arquitectura es la generación de un movimiento centrífugo desde la superficie pictórica hacia el espacio del espectador. De este modo, la denominada "perspectiva invertida" de la pintura bizantina (esto es, la proyección de los objetos de la composición hacia el espacio del espectador) se genera unitariamente desde el mismo fondo pictórico y no en cada objeto particular, como suele suceder en la pintura bizantina. En nuestra opinión, este tratamiento de la arquitectura y el paisaje contribuye a destacar la figura humana como centro de la composición, poniendo de relieve cada gesto de los personajes que conforman la escena. 


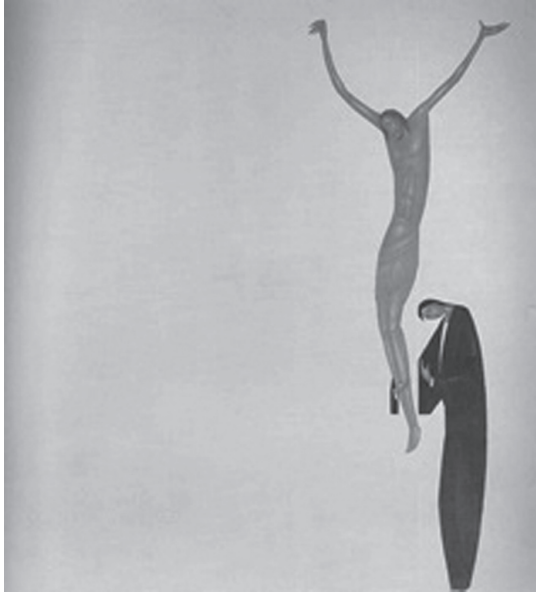

28. M. Vasilakis, La Crucificción, 2002, $(1,85 \times 1,75 \mathrm{~m})$.

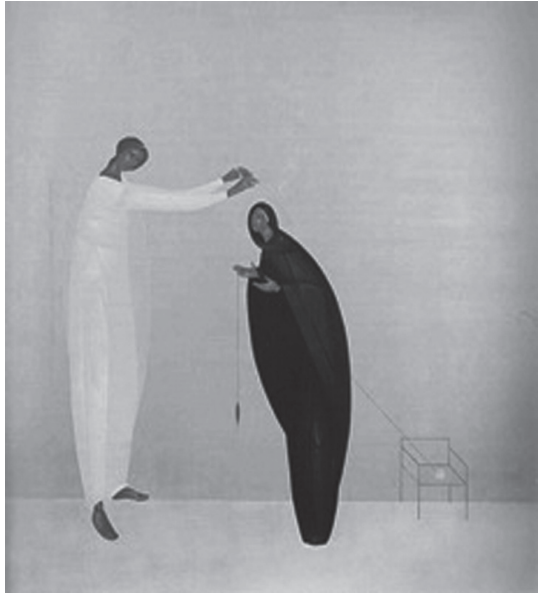

29. M. Vasilakis, La Anunciación, 2002, $(1,85 \times 1,75 \mathrm{~m})$.

Esto último se puede apreciar claramente en las figuras 28 y 29, donde los objetos son apenas insinuados en la superficie dorada, centrando toda nuestra atención en el diálogo corporal de los protagonistas de la Historia Sagrada. En este sentido, el dramatismo alcanzado en el ícono de la Crucificción es directamente proporcional a la abstracción que presenta, insinuando la forma de la Cruz con el cuerpo moribundo de Cristo y el abrazo de la Virgen María. Esta magistral composición, nos parece, muestra la síntesis alcanzada por Vasilakis entre las dos tendencias que mencionábamos más arriba: una aguda geometrización de la arquitectura y el paisaje en contraste con la figura humana donde esta voluntad de abstracción aparece más tímidamente. Para armonizar ambas tendencias, además de las operaciones compositivas que hemos mencionado, Vasilakis sustituirá los contrastes de colores por una atmósfera cromática unificada de colores cálidos, en plena armonía con los fondos dorados.

Como señalamos más arriba, para realizar este paso definitivo hacia una síntesis, Vasilakis recurrirá a movimientos pictóricos del siglo XX. Así, en el tratamiento predominantemente geométrico del fondo que se funde con la arquitectura y el paisaje, se pueden reconocer elementos del Suprematismo ruso, corriente pictórica íntimamente vinculada a la pintura bizantina ya en la obra de Kazimir Malevic (1879-1935), pintor ruso de principios del siglo XX. Por su parte, para el tratamiento de la figura humana, que hasta las obras 
de los 90 todavía se resiste a la abstracción presente en otros elementos de la composición, Vasilakis recurrirá a la obra del destacado pintor Giánnis Móralis (1916-2009), uno de los principales representantes de la pintura griega del siglo XX. Así, en la obra de Móralis, Vasilakis reconocerá un procedimiento de abstracción geométrica de la figura humana que, sin renunciar a la figuración, apunta a condensar toda la expresión del cuerpo en formas geométricas simples que se funden con la disposición geométrica del fondo pictórico. Este elemento de la pintura de Móralis en combinación con la abstracción cromática de Teófanes el griego que comentamos anteriormente, permitirá a Vasilakis armonizar el relativo naturalismo de los cuerpos bizantinos con el riguroso principio de abstracción geométrica presente en el resto de la composición.

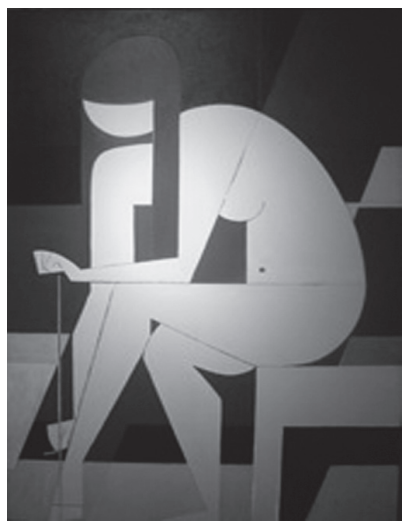

30. G. Móralis, Niña que desata su sandalia, 1973.

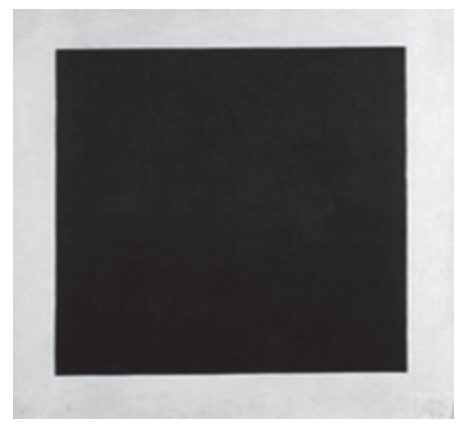

32. K. Malevic, Cuadrado negro sobre fondo blanco, 1915.

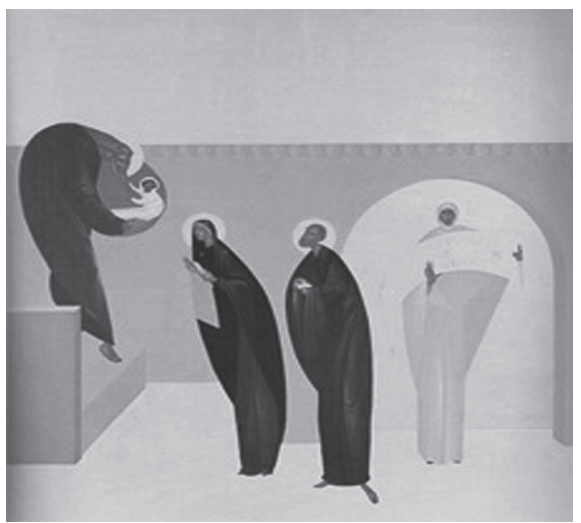

31. M. Vasilakis, La Presentación en el templo, 2002, (1,85 x 1,75 m).

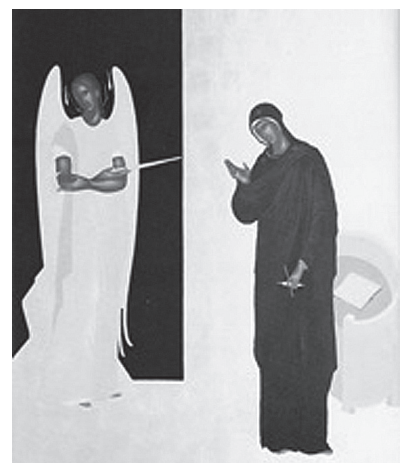

33. M. Vasilakis, La Anunciación, 1991-4. 


\section{Epílogo: los desafíos hermenéuticos de la obra de Vasilakis.}

Antes de terminar nuestro breve recorrido por la obra de Michalis Vasilakis querríamos destacar los desafíos que plantea la interpretación de una obra de esta naturaleza, tarea en gran medida pendiente. Como hemos subrayado, el camino de Vasilakis hacia la abstracción comienza a partir del reconocimiento de esta tendencia en la tradición pictórica bizantina, tradición en la que se forma como pintor por más de treinta años. Sin embargo, y en plena consonancia con su comprensión dinámica de la tradición, Vasilakis no dudará en recurrir a la pintura de nuestra época para llevar a cabo su propuesta plástica $\mathrm{y}$, de este modo, establecer un puente entre tradición y modernidad, una de las cuestiones más acuciantes de la discusión estética contemporánea. El problema de la tradición es, pues, en gran medida el problema de la modernidad, problema relacionado con la continuidad histórica de la experiencia. Ahora bien, para plantear el asunto en cuestión es necesario superar la idea decimonónica de la tradición como mero canon y retrotraerla a su significado originario, es decir, como núcleo vivo de la cultura donde se realiza toda experiencia histórica.

Por su parte, también se hace indispensable liberar la idea de modernidad de la dictadura de lo nuevo y hacernos cargo de los auténticos desafíos que plantea dicho fenómeno histórico, los cuales están relacionados con la necesidad de comprender los hechos de la historia y la cultura como una actualidad, y no sólo como información catalogable en archivos y museos. Así, la restitución de la tradición del ícono en el contexto Grecia moderna se convierte en un claro signo de esta necesidad de resituarnos en la historia. Esta necesidad, que nos convoca hacia la sabiduría del pasado y la creatividad del futuro simultáneamente, ha sido planteada por diversos filósofos a partir de la segunda mitad del siglo XX. En nuestra opinión, el gran interés que despierta la tradición del ícono en todo Occidente responde justamente a esta inquietud de nuestra época, pues, como destaca el filósofo francés Jean-Luc Marion, la tradición del ícono, "no se limita solamente, ni de entrada, a ser un punto de la historia de las ideas, ni tampoco una decisión del dogma cristiano: formula sobre todo una alternativa, y quizá la única, ante el desastre contemporáneo de la imagen" (Marion: 2006, 153). 


\section{REFERENCIAS BIBLIOGRÁFICAS}

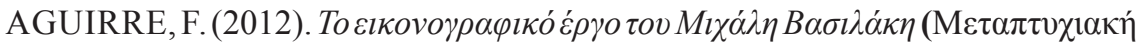

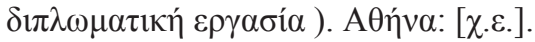

CÉZANNE, P. (1991). Correspondencia. Madrid: Visor.

CHATZIDAKIS, M. (1974) «Le Débuts de l'École Crétoise et la Question de l'École

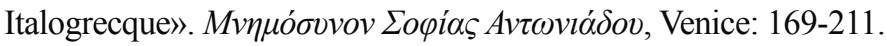

BELTING, H. (2009). Imagen y culto: una historia de la imagen anterior a la edad del arte. Madrid: Akal, 2009.

GIBBON, E. (1993). The decline and fall of the Roman Empire. New York: A.A. Knopf.

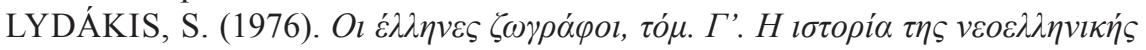

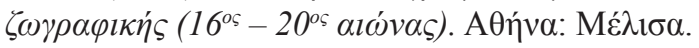

MARION, J. (2006). El cruce de lo visible. Castellón: Ellago.

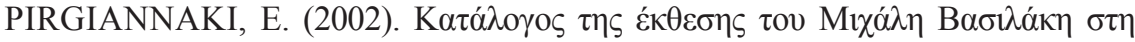

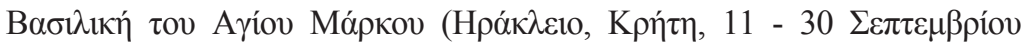

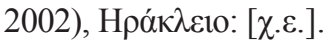

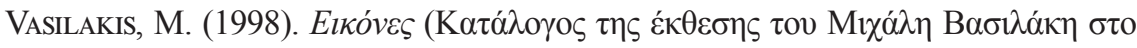

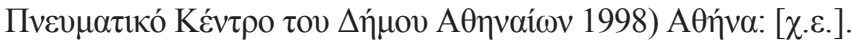

